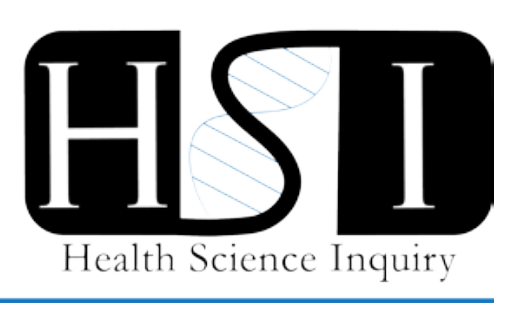

\title{
Oral health for a healthy mind: the unexplored links between oral health and dementia
}

\author{
Ricardo Alchini ${ }^{1,2}$, Túlio Eduardo Nogueira ${ }^{3}$ \\ ${ }^{1}$ Integrated Program in Neuroscience, McGill University; ${ }^{2}$ Faculty of Dentistry, McGill University; ${ }^{3}$ Department of Oral \\ Health and Society, McGill University.
}

"Regular visits to the dentist will keep our teeth healthy." Who has never heard of this recommendation? Still, we often overlook the connection between the mouth and the rest of the body. One such example is the association between oral health and dementia. Dementia is an ailment that chronically impacts the cognitive function of over 46 million patients worldwide [1]. The decline in memory and reasoning caused by dementia can be devastating to its sufferers as well as to families and caregivers of patients afflicted with the condition. As the number of dementia cases nearly doubles every 20 years, the burden to healthcare systems is coming to light. Early diagnosis of dementia-related disorders can help to alleviate some of the symptoms and slow down its progression; hence there is relevance in finding tools that detect dementia and prevent its development. Thus, we will discuss the possible impacts of oral diseases on cognitive function, and potential uses for the screening of dementia.

Several recent studies have attempted to summarize the evidence that correlates oral health conditions with dementia [2-5]. The importance of adequate mental health on the drive and ability to care for oneself is somewhat intuitive. In this context, a deterioration in oral hygiene can be an early sign of dementia. Daly et al. found that this general trend was ca observed in a variety of studies, in which dementia patients were at significantly higher risks of developing gum disease of varied intensities, cavities and tooth loss [4]. The authors, however, were conservative in suggesting that dementia caused oral disease, due to the scarcity of well-designed cohort studies on the subject, as well as to the few studies that show no correlation between impaired cognitive function and the above-mentioned oral conditions.

Oral health problems have been implicated as risk factors in the development and progression of dementia. Although less obvious, this association has been explained through different mechanisms, including the level of masticatory stimulation impacting on the cerebral blood flow and the release of inflammatory signaling molecules from localized oral inflammation into the organism, which could lead to neurodegeneration. In addition, decreased brain stimuli caused by missing teeth may reduce cerebral activation, affecting brain function and leading to the development of dementia [2].

Recent systematic reviews showed somewhat contradictory conclusions regarding the connection between tooth loss and dementia. They ranged from identifying positive association [2, 3], in which tooth loss resulted in approximately $20 \%$ to $50 \%$ increase in the risk of developing dementia, to suggesting no association at all [5]. Although most current literature positively associates tooth loss with dementia, the quality of this evidence is arguable [3, 4]. Diverse confounding factors, such as living conditions, access to dental care, dietary changes caused by tooth loss, level of dexterity, and communication ability across dementia patients, play a major role in hampering the validity of these studies. A promising approach is the Hisayama study, which followed the elderly population of a Japanese town over time [6]. Using neuroimaging, they expected to detect traces of cognitive decline as that population aged independent of behavioural changes. According to their criteria, $11.5 \%$ of the individuals developed dementia. Their findings indicated that people with 20 teeth or less were 1.6 times more likely to develop dementia than those with 20 teeth or more. Further studies with varied populations could be conducted to unveil the effect of tooth loss on the maintenance of healthy cognition.

Based on this potential relationship between dementia and oral health, we believe in the necessity of developing strategies to contain a self-feeding cycle of dementia and oral diseases. In this model, poor oral health and tooth loss contribute to dementia, which in turn deteriorate the oral condition of patients and further aggravate their mental disorder (Figure 1). Dental care must be included and provided for people with dementia and mild cognitive impairment, with the aim of improving oral health throughout life and minimizing the impact on their quality of life. A recent study assessed the current access to dental prevention advice and the care of patients diagnosed with early-stage dementia [7]. An oral education program was developed to assist nurses and other caregivers on the 


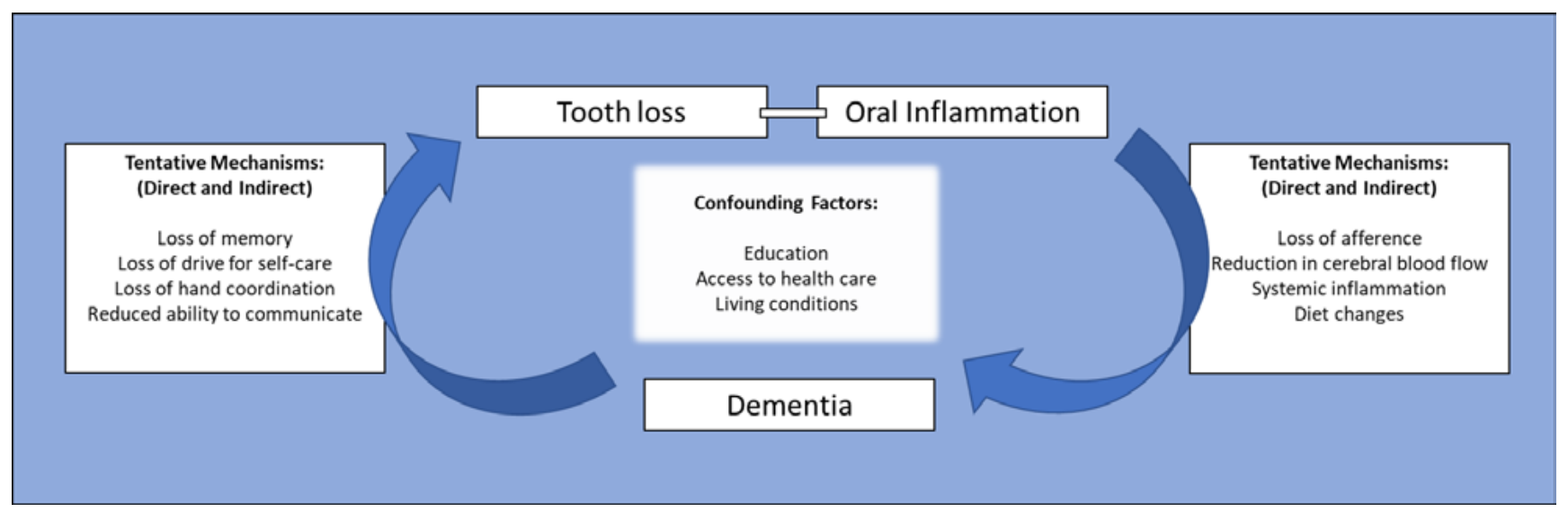

Figure 1: Proposed model. Oral conditions, such as tooth loss and oral inflammation, partially cause or exacerbate the mental disorder (dementia) of the individual, via physiological and social mechanisms. This impaired cognitive function results in deficiencies in oral care that worsen the oral condition. As a result, there is a repeated cycle in which mental and oral health continuously affect each other. Factors such as education, access to the health care system and living conditions can independently influence both health aspects (oral and mental).

oral care of these patients. However, a lack of awareness by health practitioners hindered preventative advice from being adequately provided.

Thus, higher quality health services can be provided following investigations into the association between oral and systemic health, and by translating such findings to the patients. Well-designed longitudinal studies can significantly contribute to determining the nature of the association between tooth loss and dementia. Finding such links may help dentists contribute to the early diagnosis of dementia, resulting in the development of strategies that will prevent it.

\section{Acknowledgement}

We would like to thank Dr. Raphael Freitas de Souza (McGill University) for his academic guidance and critical reading of this manuscript.

\section{References}

[1] Voos, T., Allen, C., Arora, M., Barber, R. M., Bhutta, Z. A., et al. (2016) Global, regional, and national incidence, prevalence, and years lived with disability for 310 diseases and injuries, 1990-2015: a systematic analysis for the Global Burden of Disease Study. Lancet, 388 (10053), 1545-1602.
[2] Cerutti-Kopplin, D., Feine, J., Padilha, D. M., de Souza, R. F., Ahmadi, M., Rompré, P., et al. (2016) Tooth loss increases the risk of diminished cognitive function: a systematic review and meta-analysis. J Dent Res Clin Transl Res.,1 (1), 10-19.

[3] Oh, B., Han, D. H., Han, K. T., Liu, X., Ukken, J., Chang, C., et al. (2018) Association between residual teeth number in later life and incidence of dementia: A systematic review and meta-analysis. BMC Geriatrics, $18(1), 48$.

[4] Daly, B., Thompsell, A., Sharpling, J., Rooney, Y. M., Hillman, L., Wanyonyi, K. L., et al. (2018) Evidence summary: the relationship between oral health and dementia. British Dental Journal. 223 (11), 846-853.

[5] Wu, B., Fillenbaum, G. G., Plassman, B. L., Guo, L. (2016) Association Between Oral Health and Cognitive Status: A Systematic Review. Journal of the American Geriatrics Society. 64 (8), 1752.

[6] Takeuchi, K., Ohara, T., Furuta, M., Takeshita, T., Shibata, Y., Hata, J., et al. (2017) Tooth Loss and Risk of Dementia in the Community: the Hisayama Study. Journal of the American Geriatrics Society, 65 (5), e95e100.

[7] Emanuel, R., Sorensen, A. (2018) A study of oral health prevention behaviours for patients with early stage dementia. British Dental Journal, 224 (1), 38-42. 

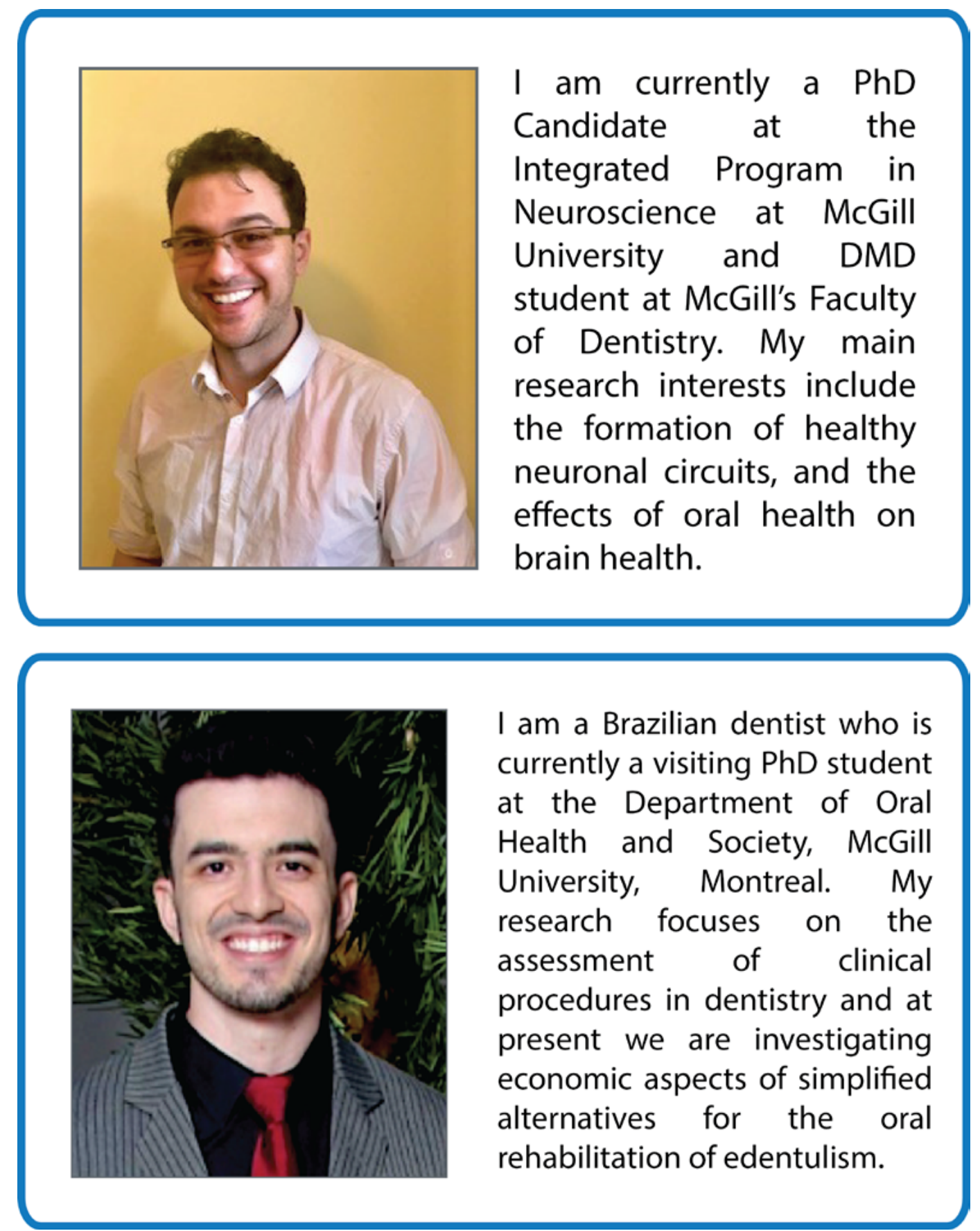